\title{
ORIGINAL ARTICLE Plasma phospholipids and fatty acid composition differ between liver biopsy-proven nonalcoholic fatty liver disease and healthy subjects
}

\author{
DWL Ma ${ }^{1}$, BM Arendt ${ }^{2}$, LM Hillyer ${ }^{1}$, SK Fung ${ }^{2,3}$, I McGilvray $^{2,3}$, M Guindi $^{2,3,4}$ and JP Allard ${ }^{2,3}$
}

BACKGROUND: There is growing evidence that nonalcoholic fatty liver disease (NAFLD) is associated with perturbations in liver lipid metabolism. Liver phospholipid and fatty acid composition have been shown to be altered in NAFLD. However, detailed profiles of circulating lipids in the pathogenesis of NAFLD are lacking.

OBJECTIVE: Therefore, the objective of the present study was to examine circulating lipids and potential mechanisms related to hepatic gene expression between liver biopsy-proven simple steatosis (SS), nonalcoholic steatohepatitis (NASH) and healthy subjects. SUBJECTS: Plasma phospholipid and fatty acid composition were determined in 31 healthy living liver donors as healthy controls (HC), 26 patients with simple hepatic steatosis (SS) and 20 with progressive NASH. Hepatic gene expression was analyzed by Illumina microarray in a subset of $22 \mathrm{HC}, 16 \mathrm{SS}$ and $14 \mathrm{NASH}$.

RESULTS: Concentrations of phosphatidylethanolamine (PE) increased relative to disease progression, $\mathrm{HC}<\mathrm{SS}<\mathrm{NASH}$ $\left(170<210<250 \mathrm{~g} \mathrm{ml}^{-1}\right)$, and was significantly different $(P<0.05)$ between $\mathrm{HC}$ and NASH. Circulating phosphatidylserine (PS) and phosphatidylinositol were higher in SS and NASH compared with HC $(P<0.05)$, but there was no difference between SS and NASH. Fatty acid composition of phospholipids was also remodeled. In particular, docosahexaenoic and arachidonic acid were higher $(P<0.05)$ in SS and NASH relative to HC in PS. Differentially expressed hepatic genes included ETNK1 and PLSCR1 that are involved in PE synthesis and PS transport, respectively.

CONCLUSIONS: The present study demonstrates that there is a disruption in phospholipid metabolism that is present in SS, but more pronounced in NASH. Intervention studies targeted at lipid metabolism could benefit SS and NASH.

Nutrition \& Diabetes (2016) 6, e220; doi:10.1038/nutd.2016.27; published online 18 July 2016

\section{INTRODUCTION}

Obesity and the development of associated diseases such as cardiovascular disease, type 2 diabetes and nonalcoholic fatty liver disease (NAFLD) continue to be the leading causes of morbidity and mortality. Thus, identifying factors for the pathogenesis of obesity and associated diseases at an early stage are urgently needed.

NAFLD is a growing health problem, as it can lead to liver cirrhosis, end stage liver disease or hepatocellular carcinoma in some patients. Perturbations in phospholipid and fatty acid composition have been identified in mouse models and human patients with NAFLD, ${ }^{1-3}$ but more precise knowledge about perturbations in lipid metabolism could facilitate the development of effective new treatments for NAFLD. We have previously shown that the hepatic phosphatidylcholine (PC) to phosphatiylethanolamine $(P E)$ ratio is decreased ${ }^{4}$ and that there are marked changes in essential fatty acid composition in the liver of NAFLD patients. ${ }^{1,5}$ Altered PC/PE ratio was also observed in red blood cells, ${ }^{4}$ but this parameter was not measured in plasma. Plasma lipids under fasting conditions reflect mainly lipids and fatty acids exported from the liver, thus may also be potential biomarkers of altered liver lipid metabolism. Furthermore, a growing number of lipid classes have been identified in plasma that may have utility as biomarkers of disease risk. These include phosphatidylserine (PS), lyso-PC and sphingomyelin (SM), which have been associated with inflammation and cellular apoptosis. ${ }^{6-8}$ Therefore, the objective of the present study was to determine whether there are differences in circulating plasma phospholipid species between patients with simple hepatic steatosis (SS) or the progressive nonalcoholic steatohepatitis (NASH) and healthy living liver donors as healthy controls (HC). Correlations with hepatic gene expression pertaining to phospholipid metabolism were also examined to explore potential mechanisms of action.

\section{MATERIALS AND METHODS}

Patients

This was a cross-sectional study. Between October 2006 and February 2009 , male and female ambulatory patients (age $\geqslant 18$ years) attending the liver clinic and healthy donors from the Living Donor Program for liver transplant recipients at the University Health Network, Toronto, Canada, were enrolled. From the liver clinic, we recruited patients with elevated liver enzymes (aspartate transaminase and/or alanine transaminase at least 1.5 times upper limit of normal) and suspected NAFLD undergoing diagnostic liver biopsy. Exclusion criteria: alcohol consumption $>20 \mathrm{~g} \mathrm{~d}^{-1}$; liver disease

\footnotetext{
${ }^{1}$ Department of Human Health and Nutritional Sciences, College of Biological Science, University of Guelph, Guelph, Ontario, Canada; ${ }^{2}$ Toronto General Hospital, University Health Network, Toronto, Ontario, Canada; ${ }^{3}$ Department of Medicine, University of Toronto, Toronto, Ontario, Canada and ${ }^{4}$ Department of Pathology and Laboratory Medicine, Cedars-

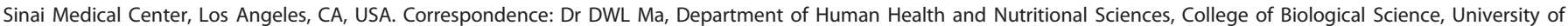
Guelph, Animal Science/Nutrition Building, Room 342, 491 Gordon Street, Guelph, Ontario, Canada N1G 2W1 or Dr JP Allard, Department of Medicine, University Health Network, 585 University Avenue, Suite 9NU-973, Toronto, Ontario, Canada M5G 2 C4.
} 
of any etiology other than NAFLD (for example, viral hepatitis, autoimmune hepatitis and Wilson's disease); anticipated need for liver transplantation within 1 year or complications of liver disease; any reasons contraindicating a liver biopsy; medications known to precipitate steatohepatitis, supplementation of antioxidant vitamins, ursodeoxycholic acid or any experimental drug in the 6 months prior to entry; pregnancy or lactation. Controls were healthy living donors meeting the criteria as per transplantation protocol for the Living Donor Program. In addition, the same exclusion criteria as for NAFLD applied. Donors were recruited shortly before the planned transplantation. Each participant provided one fasting blood sample. Liver samples for histological assessment of NAFLD were obtained during a percutaneous needle biopsy for patients. Presence of a healthy liver in HC (no steatosis or cirrhosis) was confirmed by ultrasound, transient elastography, computed tomography and/or magnetic resonance imaging. In case of any doubt, a liver biopsy was performed. A wedge biopsy was taken for histology in all donors at the time of partial hepatectomy. Hepatic gene expression was measured in all groups and reported separately. ${ }^{5}$ The study was performed according to the guidelines of the 1975 Declaration of Helsinki and was approved by the Research Ethics Board, University Health Network, Toronto, Ontario, Canada (REB \#03-0505-A, 8 October 2003, Amendments \#2 for healthy donors, 29 August 2007). Approval was also obtained from the Research Ethics Board, University of Guelph, Guelph, Ontario, Canada (REB\#10JN028, 9 July 2011). All subjects gave their informed written consent.

\section{Sample collection and preparation}

Liver tissue was divided and stored in formalin for histological assessment, and in RNA preserving agent, RNAlater (Qiagen, Hilden, Germany), for gene expression analysis. Blood for phospholipid profiling was collected after overnight fast in ethylenediaminetetraacetic acid containing tubes and centrifuged $(910 \mathrm{~g}, 10 \mathrm{~min})$. Plasma was collected and frozen at $-80^{\circ} \mathrm{C}$ until analysis.

\section{Liver histology}

Two hematoxylin-eosin-stained sections for necroinflammatory grading and two Masson's trichrome-stained sections for assessment of fibrosis were reviewed. The Brunt system was used to determine the degree of steatosis, inflammation and fibrosis in NAFLD. ${ }^{9}$ Presence of steatosis was defined as having at least $5 \%$ of hepatocytes containing large-droplet fat. To distinguish between cases with SS and NASH, a separate category for zone 3 fibrosis characteristic of steatohepatitis as well as hepatocellular ballooning were evaluated. Lobular inflammation and NAFLD activity score were also assessed. ${ }^{10}$

\section{Plasma phospholipid analysis}

Lipids from plasma were extracted according to the Folch method. ${ }^{11}$ Briefly, $100 \mu \mathrm{l}$ of plasma was added to $4 \mathrm{ml}$ chloroform:methanol solution $(2: 1, \mathrm{v} / \mathrm{v})$. Samples were vortexed for $1 \mathrm{~min}$, flushed with nitrogen gas and incubated at $4{ }^{\circ} \mathrm{C}$ overnight. The following day, samples were centrifuged at $\sim 600 \mathrm{~g}$ for $10 \mathrm{~min}\left(21^{\circ} \mathrm{C}\right)$ to separate phases. The lower chloroform layer was extracted and transferred to a fresh test tube, and dried under a gentle stream of nitrogen gas. The lipid was reconstituted in $100 \mu \mathrm{l}$ of chloroform and spotted on a $2-\mathrm{cm}$ scored lane of a $20 \times 20-\mathrm{cm}$ H-plate (VWR, Mississauga, ON, Canada, Silica Gel 60 pre-coated plates). The H-plate was previously activated by heating at $100^{\circ} \mathrm{C}$ for $1 \mathrm{~h}$. Phospholipid classes including SM, lyso-PC, PC, $\mathrm{PS}$, phosphatidylinositol (PI) and phosphatidylethanolamine (PE) fractions were separated along with authentic standards in a solvent mixture of chloroform $/$ methanol $/ 2$-propanol $/ \mathrm{KCl} \quad(0.25 \% \quad \mathrm{w} / \mathrm{v}$ )/triethylamine (Fisher, Ottawa, ON, Canada and Sigma, Oakville, ON, USA) at a ratio of 30:9:25:6:18 (v/v). Bands were visualized under ultraviolet light after lightly spraying with 8-anilino-1-naphthalene sulfonic acid $(0.1 \% \mathrm{w} / \mathrm{v}$, Fluka, Oakville, ON, USA). Bands were scraped off the plate and transferred to a test tube containing a known amount of heptadecanoic acid (17:0). Lipids were converted to fatty acid methyl esters with the addition of $14 \%$ boron trifluoride in methanol (Sigma) and incubated at $100^{\circ} \mathrm{C}$ for $1 \mathrm{~h}$. Fatty acid methyl esters were quantified on an Agilent $6890 \mathrm{~N}$ gas chromatograph equipped with flame ionization detection (Mississauga, ON, Canada) and separated on an Supelco SP-2560 fused-silica capillary column $(100 \mathrm{~m}$, $0.2 \mu \mathrm{m}$ film thickness, $0.25 \mathrm{~mm}$ internal diameter; Sigma). Samples were injected in splitless mode. The injector and detector ports were set at $250^{\circ} \mathrm{C}$. Fatty acid methyl esters were eluted using a temperature program set initially at $60^{\circ} \mathrm{C}$ and held for $2 \mathrm{~min}$, increased at $13^{\circ} \mathrm{C}$ per min and held at $170{ }^{\circ} \mathrm{C}$ for $4 \mathrm{~min}$, increased at $6.5^{\circ} \mathrm{C}$ per min to $175{ }^{\circ} \mathrm{C}$, increased at $2.6^{\circ} \mathrm{C}$ per min to $185^{\circ} \mathrm{C}$, increased $1.3^{\circ} \mathrm{C}$ per min to $190^{\circ} \mathrm{C}$, and finally increased $13^{\circ} \mathrm{C}$ per min to $240^{\circ} \mathrm{C}$ and held for $13 \mathrm{~min}$. The run time per sample was $37.77 \mathrm{~min}$. The carrier gas was hydrogen, set to a $30 \mathrm{ml}$ per min constant flow rate. Peaks were identified by comparing with retention times of fatty acid methyl ester standards (Nu-Chek-Prep, Elysian, MN, USA) using EZchrom Elite version 3.2.1 software (Mississauga, ON, Canada). Quantitative amounts of phospholipid classes were calculated by proportional comparison of gas chromatography peak areas of total fatty acids with that of the 17:0 internal standard. Fatty acid percent composition was determined relative to total peak area.

\section{Analysis of hepatic gene expression by microarray}

The analysis of hepatic gene expression has been described in detail previously ${ }^{5}$ and only genes related to phospholipid processes were examined for the present study. Briefly, total RNA from liver biopsies was extracted (mirVanaTM miRNA Isolation kit, Life Technologies Corp., Carlsbad, CA, USA), and RNA concentration, purity and quality were measured. Two hundred nanograms RNA were used for analysis with the Whole Genome Gene DASL HT Assay (Illumina Inc., San Diego, CA, USA) and the Human HT-12 V4 BeadChip (Illumina Inc.). Data were controlled for quality and analyzed using GeneSpring v12.5 (Agilent).

Differentially expressed genes (DEG) were identified by one way analysis of variance with a Benjamini-Hochberg false-discovery rate (FDR) $q<0.05$ and Tukey's HSD post hoc test. DEG were filtered for at least twofold up or downregulation. We searched the list of DEG among the groups for genes related to phospholipid metabolism. Expression levels (normalized but not median-centered and log2-transformed) for phospholipid-related DEG were then used for correlation analyses.

\section{Statistical analysis}

Data are presented as mean \pm s.d., median (25th; 75th percentile) or proportion of patients. The experimental data were subjected to a Wilcoxon test (continuous demographic and clinical data), Fisher's exact test (categorical data) or analysis of variance (phospholipid data) for discernment of statistical differences using a predetermined upper limit of probability of $P<0.05$. Multiple comparison testing of sample means was performed by the Duncan's multiple range test. The analysis was performed using the SAS system for Windows version 9.2 (SAS Institute Inc., Cary, NC, USA). The required sample size to detect a difference in means of $40 \mathrm{\mu g} \mathrm{ml}^{-1}$ (with a s. d. of $15 \mathrm{\mu g} \mathrm{ml}^{-1}$ ) in $\mathrm{Pl}$, the smallest fraction (Figure 1) at $90 \%$ power is $n=9$.

\section{RESULTS}

\section{Subjects}

A total of 31 HC, 26 SS and 20 NASH plasma samples were analyzed. The SS group included three individuals who were recruited as healthy liver donors, but were found to have $5-10 \%$ steatosis on liver biopsy. Counting patients with a known diagnosis of diabetes or $\mathrm{HbA} 1 \mathrm{c} \geqslant 0.065$ or fasting glucose $\geqslant 7.0 \mathrm{mmol} \mathrm{I}^{-1}$ : HC: none, SS: 5/26 (19.2\%), NASH: $5 / 20$ (25\%). All other patient and clinical characteristics for patients and controls are reported in Tables 1 and 2.

\section{Plasma phospholipids}

Quantitative levels of plasma phospholipid species were determined from fasted blood samples. Plasma concentrations of PI and PS were higher in SS and NASH patients compared with HC (Figure 1), whereas PE was highest in NASH followed by SS and HC.

\section{Fatty acid composition of plasma phospholipids}

The percent fatty acid composition was determined in each of the plasma phospholipid classes. The major $n-6$ and $n-3$ fatty acids are reported in Table 3. The essential fatty acids, linoleic acid and a-linolenic acid, and their long-chain polyunsaturated products arachidonic acid, eicosapentaenoic acid and docosahexaenoic acid (DHA) have an important role as substrates for the synthesis of pro and anti-inflammatory eicosanoids. Significant $(P<0.05)$ differences for one or more of these fatty acids were found in all phospholipid classes. In particular, DHA was higher in SS and/or NASH patients relative to $\mathrm{HC}$ in several phospholipid species, with the largest differences (threefold and fivefold increase in SS and 
a

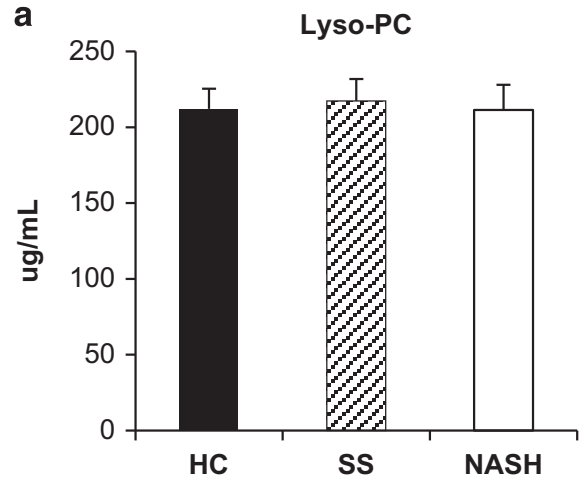

C

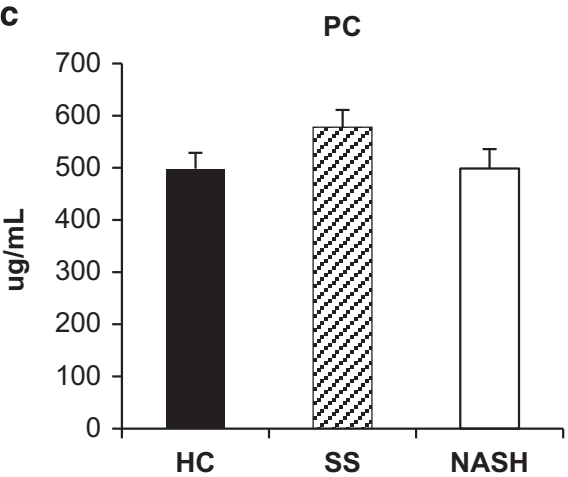

e

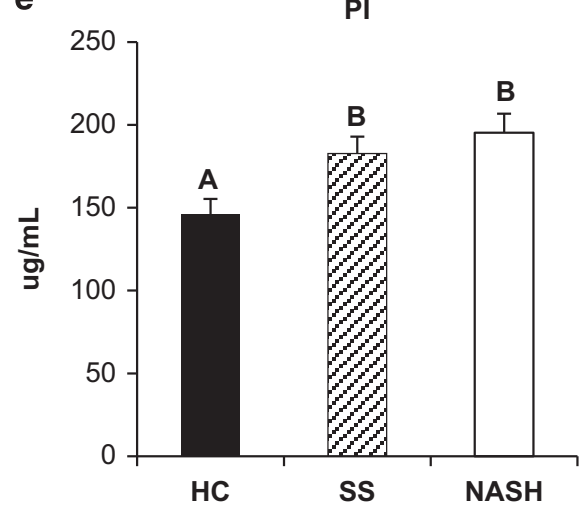

b

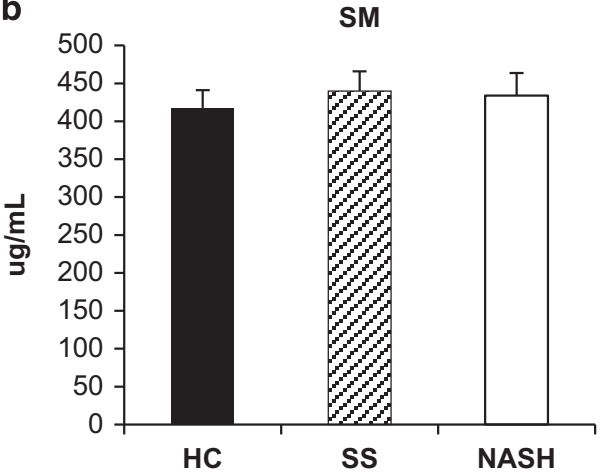

d

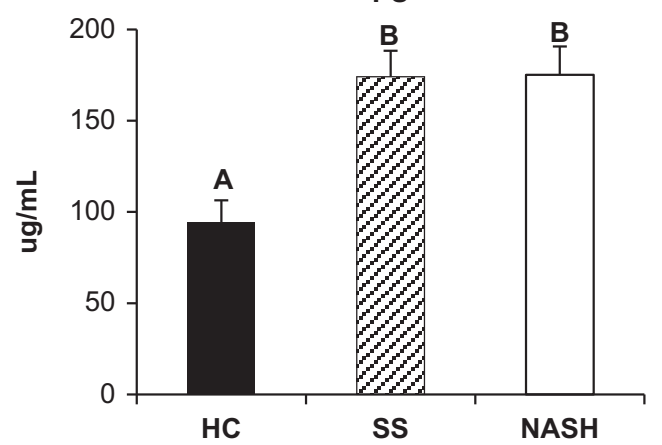

f

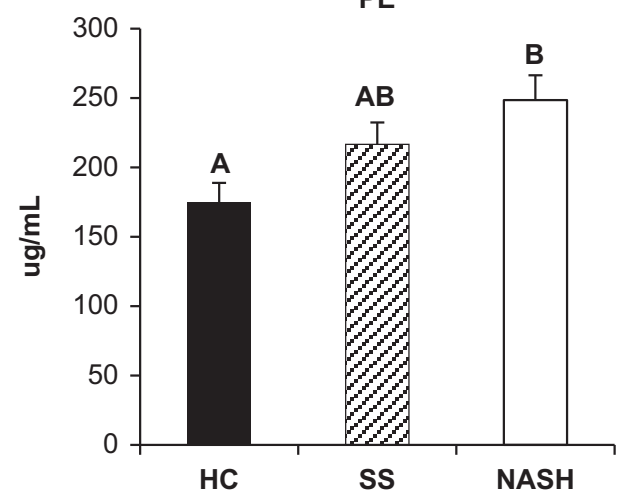

Figure 1. Lipid concentrations found in plasma phospholipid fractions ( $\mu \mathrm{g}$ fatty acid per $\mathrm{ml}$ of plasma) determined by gas chromatography for control (HC), SS and NASH subjects. (a) Lyso-phosphotidylcholine, $P=0.95$. (b) Sphingomyelin, $P=0.81$. (c) Phosphotidylcholine, $P=0.08$. (d) Phosphotidylserine, $P<0.0001$. (e) Phosphotidylinositol, $P=0.0024$. (f) Phosphotidylethanolamine, $P=0.006$. Bars represent means \pm s.d. $(n=31 \mathrm{HC}, 26 \mathrm{SS}$ and $20 \mathrm{NASH})$. Bars not sharing a letter are significantly different $(P<0.05)$ by Duncan's new multiple range test.

$\mathrm{NASH}$, respectively) in $\mathrm{PI}$ and $\mathrm{PS}$. Arachidonic acid was also higher in SS and NASH patients relative to HC in PS. In contrast, arachidonic acid in PE was lower in NASH than in $\mathrm{HC}$ and SS. Differences were also observed for linoleic acid in lyso-PC (lower in $\mathrm{SS}$ and NASH than in $\mathrm{HC}$ ) and SM (higher in NASH than in HC). The changes in single fatty acids also lead to altered $n 3 / n 6$ ratios in all phospholipid classes.

Hepatic gene expression

Among the patients ( $31 \mathrm{HC}, 26 \mathrm{SS}$ and $20 \mathrm{NASH})$ that provided plasma for analysis, 52 (22 HC, 16 SS and 14 NASH) of these study participants also provided tissue for hepatic gene expression analysis as part of our previous study. ${ }^{5}$ There was no statistically significant difference between these patients and those who did not have gene expression measured (diagnosis, demography and clinical data) except for fasting glucose, which was slightly higher in those who had gene expression assessed (median (25th; 75th percentile)); $\left(5.2(4.7 ; 5.8)\right.$ versus $4.9(4.5 ; 5.2) \mathrm{mmol} \mathrm{I}^{-1}$; $P=0.0358$ ). Microarray data are presented in Table 4. Several genes related to phospholipid metabolism were differentially expressed between HC, SS and NASH in current participants. Correlations between these genes and plasma phospholipids are shown in Table 4.

\section{DISCUSSION}

We have shown that specific plasma phospholipids differ between $\mathrm{HC}$, SS and NASH. We also found that these changes are associated with changes in the expression of genes involved in phospholipid metabolism in the liver. These findings suggest that in NAFLD, there is a disruption in phospholipid metabolism that is already present in SS, but more pronounced in NASH compared with $\mathrm{HC}$. 
Table 1. HC, SS and NASH patient obesity and hepatic steatosis

\begin{tabular}{|c|c|c|c|c|}
\hline Characteristic & $\begin{array}{c}H C \\
\text { Percentage of patients }(\mathrm{n} / \mathrm{N})\end{array}$ & $\begin{array}{c}\text { SS } \\
\text { Percentage of patients }(\mathrm{n} / \mathrm{N})\end{array}$ & $\begin{array}{c}\mathrm{NASH} \\
\text { Percentage of patients }(\mathrm{n} / \mathrm{N})\end{array}$ & P-value \\
\hline $\begin{array}{l}\text { Obesity status } \\
\text { Normal weight } \\
\text { Overweight } \\
\text { Obesity }\end{array}$ & $\begin{array}{l}41.9(13 / 31) \\
45.2(14 / 31) \\
12.9(4 / 31)\end{array}$ & $\begin{array}{l}26.9(7 / 26) \\
46.2(12 / 26) \\
26.9(7 / 26)\end{array}$ & $\begin{array}{l}15.0(3 / 20) \\
20.0(4 / 20) \\
65.0(13 / 20)\end{array}$ & $P<0.05$ \\
\hline $\begin{array}{l}\text { Gender } \\
\text { Female } \\
\text { Male }\end{array}$ & $\begin{array}{l}51.6(16 / 31) \\
48.4(15 / 31)\end{array}$ & $\begin{array}{l}26.9(7 / 26) \\
73.1(19 / 26)\end{array}$ & $\begin{array}{l}55.0(11 / 20) \\
45.0(9 / 20)\end{array}$ & NS $(P=0.10)$ \\
\hline $\begin{array}{c}\text { Steatosis grading } \\
0 \\
1 \\
2 \\
3\end{array}$ & $\begin{array}{l}100(31 / 31) \\
0.0(0 / 31) \\
0.0(0 / 31) \\
0.0(0 / 31)\end{array}$ & $\begin{array}{l}0.00(0 / 26) \\
65.4(17 / 26) \\
19.2(5 / 26) \\
15.4(4 / 26)\end{array}$ & $\begin{array}{r}0.0(0 / 20) \\
30.0(6 / 20) \\
35.0(7 / 20) \\
35.0(7 / 20)\end{array}$ & $P<0.05$ \\
\hline
\end{tabular}

Abbreviations: $0,<5 \%$ steatosis in $\%$ of hepatocytes involved; $1,5-33 \%$ steatosis; $2,33-66 \%$ steatosis; $3,>66 \%$ steatosis; HC, healthy controls; NASH, nonalcoholic steatohepatitis; NS, not significant; SS, simple steatosis. ${ }^{a}$ Steatosis grading according to Brunt et al. ${ }^{9}$

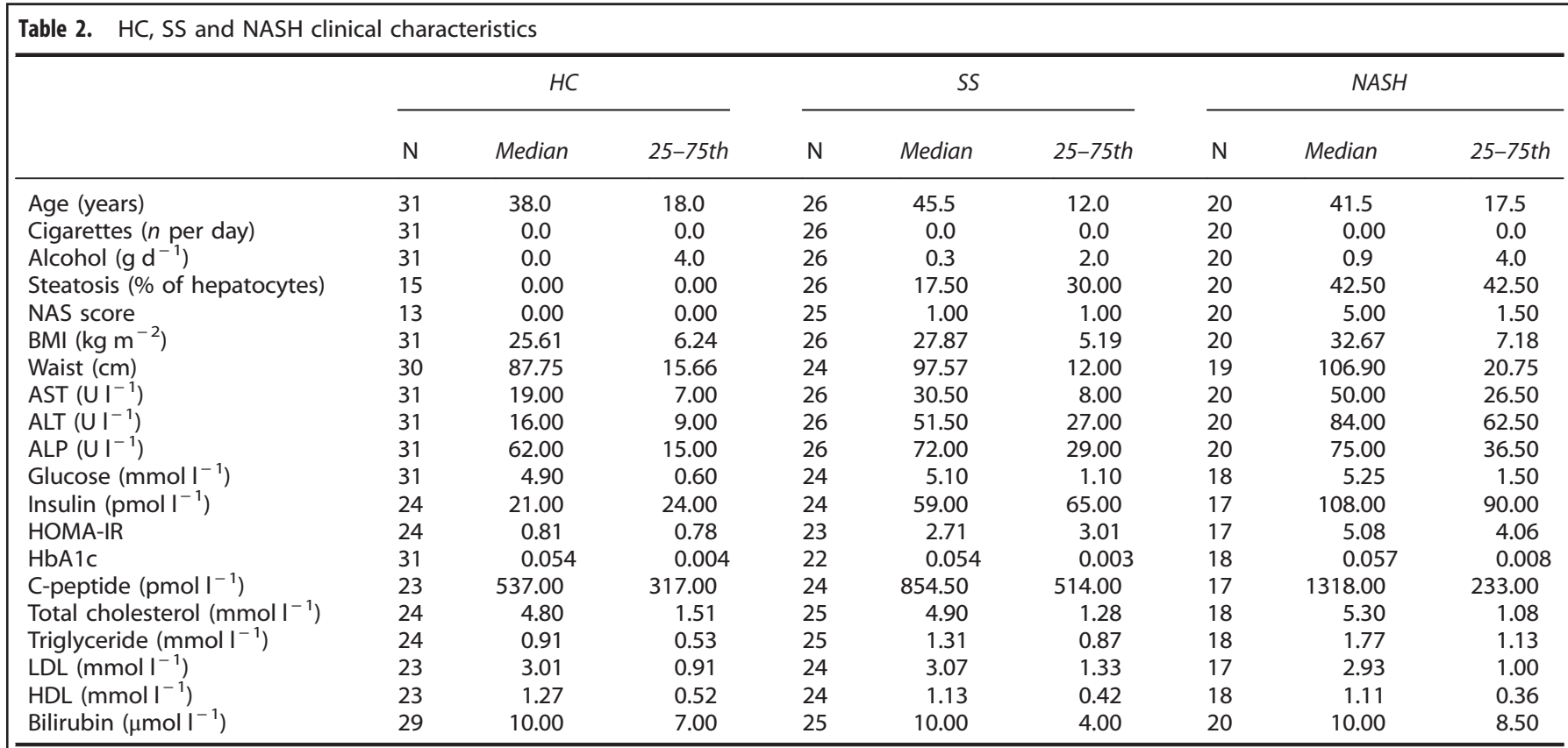

Abbreviations: ALP, alkaline phosphatase; ALT, alanine transaminase; AST, aspartate transaminase; BMl, body mass index; HbA1c, hemoglobin A1c; HDL, high-density lipoprotein cholesterol; HOMA-IR, homeostasis model of assessment for insulin resistance; LDL, low-density lipoprotein cholesterol; NAS, nonalcoholic fatty liver disease activity score.

We showed previously that liver PC/PE ratio, a biomarker of NAFLD, was lower in patients with SS and NASH compared with HC, and there was no difference between SS and NASH. ${ }^{4}$ In the present study, we show PC was not different, but plasma PE differed slightly among patient groups that was lowest in $\mathrm{HC}$ and higher in NASH (Figure 1). Although there was a trend toward lower plasma $\mathrm{PC} / \mathrm{PE}$ ratio, where $\mathrm{HC}>\mathrm{SS}>\mathrm{NAHS}$, there was no difference among groups. These observations suggest that compensatory mechanisms act to maintain circulating plasma PC/PE ratio at the expense of lower liver phospholipids. ${ }^{12}$ This compensation has been observed in mice lacking the gene for PE methyl transferase, required for the synthesis of PC in the liver and lipoprotein transport. ${ }^{13}$ Thus, although the liver is able to compensate for impairments in phospholipid synthesis at early stages of the disease, this may not be sustainable for the long term leading to observable differences in circulating PE, observed in the more advanced stage of NAFLD, that is, NASH.

There is growing recognition that plasma contains a multitude of lipids that may have utility as biomarkers of chronic disease. In addition to PC and PE, SM, lyso-PC, PS and PI were also determined in $\mathrm{HC}$ and NAFLD patients. Although these species are not as well understood as $\mathrm{PC}$ and $\mathrm{PE}$, their presence may also have important health implications. These phospholipid classes are associated with inflammation and cellular apoptosis, and therefore may be associated with NAFLD and disease severity (SS versus NASH). ${ }^{7,8,14-18}$ PS was significantly higher $(P<0.05$, Figure 1$)$ in SS and NASH as compared with $\mathrm{HC}$, but no difference was detected between SS and NASH. PS has an important role in cellular apoptosis. Typically, it is found in the inner leaflet of the plasma membrane, but is externalized to the cell surface in response to stimuli signaling for cell death. ${ }^{6}$ We 
Table 3. Plasma phospholipid fatty acid composition

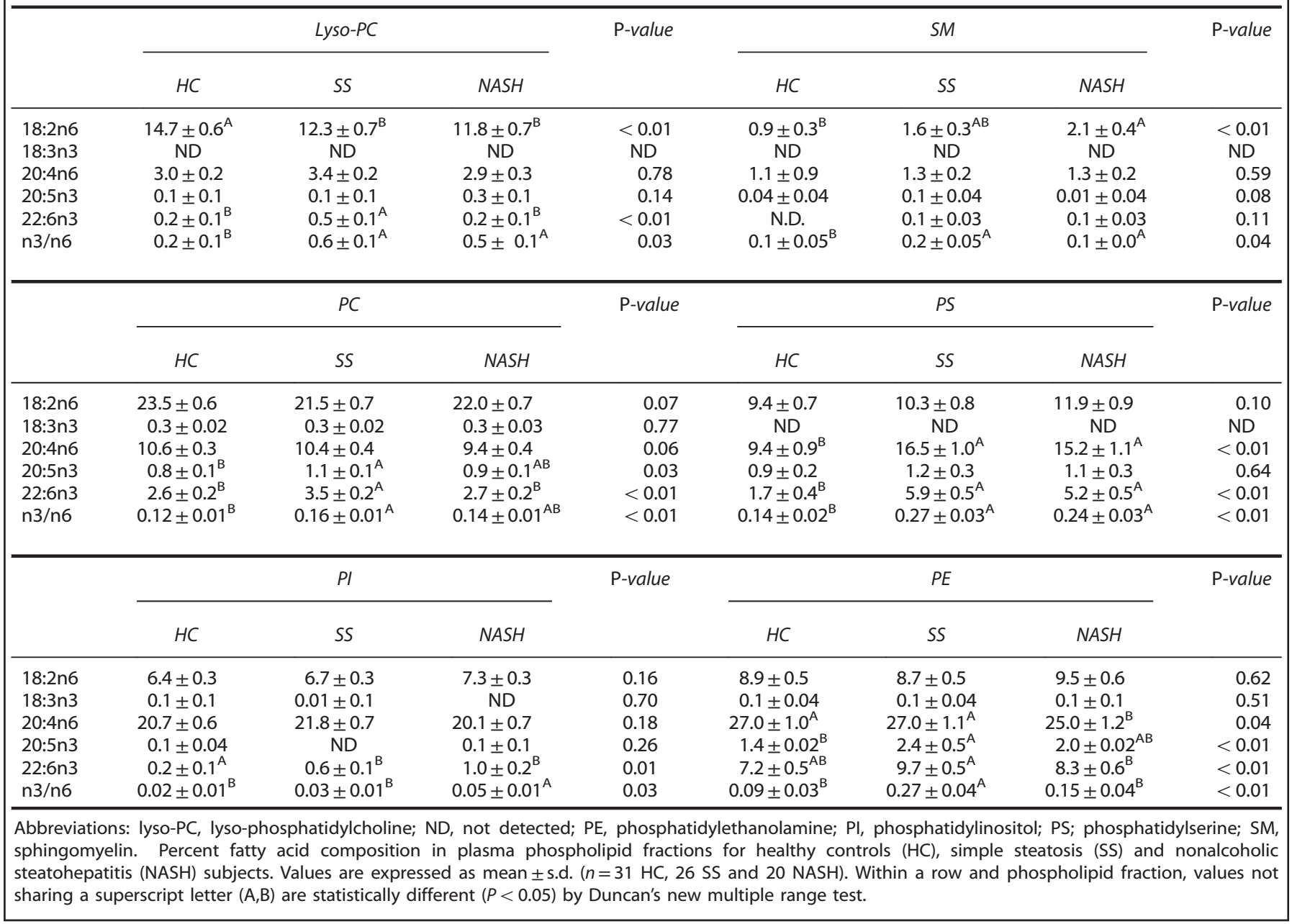

Table 4. Hepatic gene expression by microarray and the correlation of differentially expressed genes in HC, SS and NASH patients for genes with known phospholipid associated function

\begin{tabular}{|c|c|c|c|c|c|c|c|}
\hline \multirow[t]{2}{*}{ Probe ID } & \multirow[t]{2}{*}{ Entrez gene ID } & \multicolumn{3}{|c|}{ Fold change } & \multirow[t]{2}{*}{ P-value } & \multirow[t]{2}{*}{ Symbol } & \multirow[t]{2}{*}{ Synonyms } \\
\hline & & NASH vs HC & SS vs HC & NASH vs SS & & & \\
\hline \multicolumn{8}{|l|}{$P C$} \\
\hline 840678 & 5168 & 1.43 & -1.47 & 2.10 & $6.87 \mathrm{E}-05$ & ENPP2 & NPP2; ATX; LysoPLD; ATX-X; PDNP2; PD-IALPHA; FLJ26803 \\
\hline 2480338 & 1622 & 2.03 & 2.25 & NS & $6.11 \mathrm{E}-07$ & $\mathrm{DBI}$ & MGC70414; ACBD1; ACBP \\
\hline \multicolumn{8}{|l|}{$P E$} \\
\hline \multicolumn{8}{|l|}{ PS } \\
\hline 3890609 & 5359 & -1.91 & -2.15 & NS & $7.23 E-05$ & PLSCR1 & MMTRA1B \\
\hline \multicolumn{8}{|l|}{ PI } \\
\hline 360475 & 5329 & -2.09 & -2.27 & NS & $5.58 \mathrm{E}-07$ & PLAUR & CD87; UPAR; URKR \\
\hline 2120068 & 9110 & 2.05 & 2.16 & NS & $4.60 \mathrm{E}-09$ & MTMR4 & FYVE-DSP2; ZFYVE11; KIAA0647 \\
\hline
\end{tabular}

Abbreviations: $\mathrm{HC}$, healthy controls; $\mathrm{NASH}$, nonalcoholic steatohepatitis; $\mathrm{NS}$, not significant; $\mathrm{PC}$, phosphatidylcholine; $\mathrm{PE}$, phosphatidylethanolamine; $\mathrm{PI}$, phosphatidylinositol; PS; phosphatidylserine; SS, simple steatosis. Genes were selected on the basis of their association with specific phospholipid fractions. Then, correlations were determined among HC, SS and NASH patients. 
hypothesized that it could be higher in NASH versus SS, but this was not significant. In tandem, the fatty acid composition of PS was observed to have significantly higher levels of arachidonic and DHA in SS and NASH patients, relative to HC (Table 4). Arachidonic acid is liberated by phospholipase A2 for the production of eicosanoids that is involved in inflammatory processes. ${ }^{19}$ In contrast, DHA is known for its anti-inflammatory properties. Reasons for the presence of higher levels of these opposing fatty acids in PS are unclear. PI was also higher in SS and NASH patients relative to $\mathrm{HC}$. PI and related metabolites are important second messengers involved in mitogen activated protein kinase and protein kinase B (PKB/Akt) signaling pathways. ${ }^{20}$ Although low levels of PS and PI have been reported in plasma, their significance in circulation are not entirely clear, but may influence the structure and function of lipoproteins. ${ }^{21-24}$ No differences were found in lyso-PC and SM among HC, SS and NASH.

The observed changes in plasma phospholipids of PE, PS and PI in SS and NASH patients suggest alterations in phospholipid metabolism by the liver. Indeed, plasma lipids have been shown to be potential biomarkers of lipid composition in the liver and other tissues. PC containing DHA has been shown to be a marker of liver PE N-methyltransferase in human liver. $^{25}$ The omega-3 index, derived from the sum of eicosapentaenoic acid and DHA from total lipids or phospholipids of red blood cells is a marker of these fatty acids in heart tissue and cardiovascular health. ${ }^{26,27}$ Changes in fat intake in men have been associated with significant changes in plasma SM, ceramides, ether phospholipids, plasmalogens and gangliosides, and elevations in PC and $\mathrm{PI}^{28}$ Alcoholic liver cirrhosis was shown to be correlated with plasma, cholesterol, lyso-PC, PC and $\mathrm{PI}^{29}$

Corresponding to changes in circulating plasma phospholipid classes, analyses of liver gene expression identified DEGs related to phospholipid metabolism that were significantly (>twofold) over or underexpressed in NASH and SS relative to $\mathrm{HC}$ patients or between NASH and SS (Table 4). Search of PE-related genes identified ethanolamine kinase (ETNK1), which was downregulated in NASH and SS patients. ETNK1 is involved in the first step in PE synthesis, and recently identified as a gene involved in squamous cell carcinoma and gastric cancers. ${ }^{30,31}$ PI-specific genes differentially expressed included lower expression of plasminogen activator urokinase receptor (PLAUR, transcript variants 1 and 2), a glycosylphosphatidylinositol (GPI)-anchored protein, and higher expression of myotubularin-related protein 4 (MTMR4) that is involved in the PI cycle and may influence plasma cholesterol. ${ }^{32}$ The PS-related gene, scramblase 1 (PLSCR1) responsible for phospholipid membrane 'flip-flop', and maintaining membrane asymmetry and potentially for the movement of intracellular PS to the extracellular face during apoptosis was lower in SS and NASH. ${ }^{33}$ The expression of acyl-CoAbinding protein gene (DBI) involved in $\mathrm{PC}$ metabolism was higher in $\mathrm{SS}$ and NASH. ${ }^{34}$ ENPP2 or autotaxin, a lysophospholipase D enzyme involved in the production of lysophosphatidic acid (LPA) has been implicated in inflammation, and cancer was more highly expressed in NASH than $\mathrm{SS}^{35}$ These changes in gene expression provide potential new avenues for further investigation to delineate how phospholipid metabolism is involved in the development of NAFLD.

A strength of the present study is both quantitative and qualitative measurements of lipid classes and fatty acids in tandem. In addition, analyses of liver samples from true $\mathrm{HC}$ are not readily accessible. The cross-sectional nature of the present study is a limitation that does not allow us to determine whether perturbations in lipid metabolism are causal or a consequence of NAFLD. This could be elucidated in prospective cohort studies with repeated measurements of lipids in patients at risk of developing NAFLD.

In summary, the present study provides evidence that the plasma phospholipid profile of NAFLD patients differ from HC, and that these changes are already present in SS and more pronounced in NASH. This suggests that there are changes early in the disease and that interventions targeting improvement in lipid metabolism may benefit both SS and NASH. Further intervention studies are warranted to assess this.

\section{CONFLICT OF INTEREST}

The authors declare no conflict of interest.

\section{ACKNOWLEDGEMENTS}

Funding for this study was provided by the Canadian Institutes of Health Research (CIHR), grants NMD-86922 and MOP-89705.

\section{AUTHOR CONTRIBUTIONS}

JPA conceptualized and obtained research funding. DWLM and LMH performed the lipid analyses. BMA was involved in the experimental design and execution of the study. SKF, IM and MG were responsible for clinical measures and obtaining tissues. MG assessed the liver biopsy samples. All authors read and approved the final manuscript.

\section{REFERENCES}

1 Allard JP, Aghdassi E, Mohammed S, Raman M, Avand G, Arendt BM et al. Nutritional assessment and hepatic fatty acid composition in non-alcoholic fatty liver disease (NAFLD): a cross-sectional study. J Hepatol 2008; 48: 300-307.

2 Arendt BM, Mohammed SS, Aghdassi E, Prayitno NR, Ma DW, Nguyen A et al. Hepatic fatty acid composition differs between chronic hepatitis $C$ patients with and without steatosis. J Nutr 2009; 139: 691-695.

3 Arendt BM, Mohammed SS, Ma DW, Aghdassi E, Salit IE, Wong DK et al. Non-alcoholic fatty liver disease in HIV infection associated with altered hepatic fatty acid composition. Curr HIV Res 2011; 9: 128-135.

4 Arendt BM, Ma DWL, Simons B, Noureldin S, Therapondos G, Guindi M et al. Non-alcoholic fatty liver disease is associated with lower hepatic and erythrocyte ratios of phosphatidylcholine to phosphatidylethanolamine. Appl Physiol Nutr Metab 2012; 38: 334-340.

5 Arendt BM, Comelli EM, Ma DW, Lou W, Teterina A, Kim T et al. Altered hepatic gene expression in nonalcoholic fatty liver disease is associated with lower hepatic n-3 and n-6 polyunsaturated fatty acids. Hepatology 2015; 61: 1565-1578. 6 Nagata S. Apoptosis and autoimmune diseases. Ann N Y Acad Sci 2010; 1209: 10-16.

7 Nixon GF. Sphingolipids in inflammation: pathological implications and potential therapeutic targets. Br J Pharmacol 2009; 158: 982-993.

8 Oestvang J, Anthonsen MW, Johansen B. LysoPC and PAF trigger arachidonic acid release by divergent signaling mechanisms in monocytes. J Lipids 2011; 2011: 532145.

9 Brunt EM, Janney CG, Di Bisceglie AM, Neuschwander-Tetri BA, Bacon BR. Nonalcoholic steatohepatitis: a proposal for grading and staging the histological lesions. Am J Gastroenterol 1999; 94: 2467-2474.

10 Kleiner DE, Brunt EM, Van NM, Behling C, Contos MJ, Cummings OW et al. Design and validation of a histological scoring system for nonalcoholic fatty liver disease. Hepatology 2005; 41: 1313-1321.

11 Folch J, Lees M, Sloane Stanley GH. A simple method for the isolation and purification of total lipides from animal tissues. J Biol Chem 1957; 226: 497-509.

12 Li Z, Agellon LB, Allen TM, Umeda M, Jewell L, Mason A et al. The ratio of phosphatidylcholine to phosphatidylethanolamine influences membrane integrity and steatohepatitis. Cell Metab 2006; 3: 321-331.

$13 \mathrm{Li}$ Z, Agellon LB, Vance DE. A role for high density lipoproteins in hepatic phosphatidylcholine homeostasis. Biochim Biophys Acta 2007; 1771: 893-900.

14 Taylor LA, Arends J, Hodina AK, Unger C, Massing U. Plasma lyso-phosphatidylcholine concentration is decreased in cancer patients with weight loss and activated inflammatory status. Lipids Health Dis 2007; 6: 17.

15 Fuchs B, Schiller J. Lysophospholipids: their generation, physiological role and detection. Are they important disease markers? Mini Rev Med Chem 2009; 9: 368-378.

16 Hammad SM. Blood sphingolipids in homeostasis and pathobiology. Adv Exp Med Biol 2011; 721: 57-66.

17 Bikman BT, Summers SA. Sphingolipids and hepatic steatosis. Adv Exp Med Biol 2011; 721: 87-97.

18 Fox TE, Bewley MC, Unrath KA, Pedersen MM, Anderson RE, Jung DY et al. Circulating sphingolipid biomarkers in models of type 1 diabetes. J Lipid Res 2011; 52: $509-517$

19 Cojocaru M, Cojocaru IM, Silosi I. Lipoprotein-associated phospholipase A2 as a predictive biomarker of sub-clinical inflammation in cardiovascular diseases. Maedica (Buchar) 2010; 5: 51-55. 
20 Balla T. Phosphoinositides: tiny lipids with giant impact on cell regulation. Physiol Rev 2013; 93: 1019-1137.

21 Phillips GB, Dodge JT. Composition of phospholipids and of phospholipid fatty acids of human plasma. J Lipid Res 1967; 8: 676-681.

22 Serna J, Garcia-Seisdedos D, Alcazar A, Lasuncion MA, Busto R, Pastor O. Quantitative lipidomic analysis of plasma and plasma lipoproteins using MALDITOF mass spectrometry. Chem Phys Lipids 2015; 189: 7-18.

23 Camont L, Lhomme M, Rached F, Le GW, Negre-Salvayre A, Salvayre R et al. Small, dense high-density lipoprotein-3 particles are enriched in negatively charged phospholipids: relevance to cellular cholesterol efflux, antioxidative, antithrombotic, anti-inflammatory, and antiapoptotic functionalities. Arterioscler Thromb Vasc Biol 2013; 33: 2715-2723.

24 Burgess JW, Neville TA, Rouillard P, Harder Z, Beanlands DS, Sparks DL. Phosphatidylinositol increases HDL-C levels in humans. J Lipid Res 2005; 46: 350-355.

25 da Costa KA, Sanders LM, Fischer LM, Zeisel SH. Docosahexaenoic acid in plasma phosphatidylcholine may be a potential marker for in vivo phosphatidylethanolamine N-methyltransferase activity in humans. Am J Clin Nutr 2011; 93: 968-974.

26 Harris WS, Sands SA, Windsor SL, Ali HA, Stevens TL, Magalski A et al. Omega-3 fatty acids in cardiac biopsies from heart transplantation patients: correlation with erythrocytes and response to supplementation. Circulation 2004; 110: 1645-1649.

27 Harris WS, Von Schacky C. The Omega-3 Index: a new risk factor for death from coronary heart disease? Prev Med 2004; 39: 212-220.

28 Meikle PJ, Barlow CK, Mellett NA, Mundra PA, Bonham MP, Larsen A et al. Postprandial plasma phospholipids in men are influenced by the source of dietary fat. J Nutr 2015; 145: 2012-2018.

29 Meikle PJ, Mundra PA, Wong G, Rahman K, Huynh K, Barlow CK et al. Circulating lipids are associated with alcoholic liver cirrhosis and represent potential biomarkers for risk assessment. PLoS One 2015; 10: e0130346.
30 Ratovitski EA. Delta Np63 alpha - responsive microRNA modulate the expression of metabolic enzymes. Curr Pharm Biotechnol 2015; 16: 832-850.

31 Chang H, Rha SY, Jeung HC, Jung JJ, Kim TS, Kwon HJ et al. Identification of genes related to a synergistic effect of taxane and suberoylanilide hydroxamic acid combination treatment in gastric cancer cells. J Cancer Res Clin Oncol 2010; 136: 1901-1913.

32 Dolley G, Lamarche B, Despres JP, Bouchard C, Perusse L, Vohl MC. Phosphoinositide cycle gene polymorphisms affect the plasma lipid profile in the Quebec Family Study. Mol Genet Metab 2009; 97: 149-154.

33 Rysavy NM, Shimoda LM, Dixon AM, Speck M, Stokes AJ, Turner $\mathrm{H}$ et al. Beyond apoptosis: the mechanism and function of phosphatidylserine asymmetry in the membrane of activating mast cells. Bioarchitecture 2014; 4: 127-137.

34 Vock C, Biedasek K, Boomgaarden I, Heins A, Nitz I, Doring F. ACBP knockdown leads to down-regulation of genes encoding rate-limiting enzymes in cholesterol and fatty acid metabolism. Cell Physiol Biochem 2010; 25: 675-686.

35 Kondo M, Ishizawa T, Enooku K, Tokuhara Y, Ohkawa R, Uranbileg B et al. Increased serum autotaxin levels in hepatocellular carcinoma patients were caused by background liver fibrosis but not by carcinoma. Clin Chim Acta 2014; 433: $128-134$

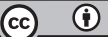

This work is licensed under a Creative Commons Attribution 4.0 International License. The images or other third party material in this article are included in the article's Creative Commons license, unless indicated otherwise in the credit line; if the material is not included under the Creative Commons license, users will need to obtain permission from the license holder to reproduce the material. To view a copy of this license, visit http://creativecommons.org/licenses/ by/4.0/

(c) The Author(s) 2016 